\title{
BILATERAL ANTERIOR GLENOHUMERAL DISLOCATION: CLINICAL CASE
}

Luís Pires Silva', Cristina Varino Sousa ${ }^{1}$, Elisa Rodrigues' ${ }^{1}$ Bruno Alpoim', Miguel Leal ${ }^{2}$

\section{ABSTRACT}

Bilateral anterior glenohumeral dislocation is a rare occurrence. We present a case of bilateral anterior glenohumeral dislocation caused by a fall. The interest in publishing this case is that this is a clinical rarity with few cases reported in the literature. An 89-year-old female patient was brought to the emergency department after a fall, complaining of intense pain in both shoulders and inability to move them. Objective examination showed clinical signs giving the suspicion of bilateral anterior glenohumeral dislocation,

\section{INTRODUCTION}

Although unilateral glenohumeral dislocation is the commonest form of joint dislocation and the type that leads the greatest number of patients to come to the Emergency Services, its bilateral form is a very rare occurrence. When this happens, posterior bilateral dislocation of the humeral head is more frequent than the anterior form or the inferior form (which is the rarest). Cases of bilateral glenohumeral dislocation have been described originating from convulsive crises (epilepsy or hypoglycemia), electrocution, falls, traction forces or muscle training exercises.

\section{CLINICAL CASE}

The patient was an 82-year-old woman who came to the Emergency Service after a posterior fall. She complained of intense pain in both shoulders, accompanied by functional incapacity. According to her description, she slipped and fell backwards, and broke her fall by bearing her weight on both hands on which was confirmed by $\mathrm{x}$-ray imaging. Both dislocations were successfully reduced in the emergency department using the modified Milch technique. When a synchronous and symmetrical force has acted on both shoulders and these are painful with significant functional limitation, the suspicion of bilateral glenohumeral dislocation is a differential diagnosis to be considered, even though it is rare.

Keywords - Shoulder Dislocation/diagnosis; Shoulder Dislocation/etiology; Shoulder Dislocation/therapy; Female; Adults; Aged

the ground. She said that she had not been suffering from loss of consciousness, epilepsy, diabetes or other pathological antecedents of relevance. On objective examination, both upper limbs were seen to present external rotation with slight abduction. Anterior tumefaction was observed (humeral head) and sulcus sign was present in both shoulders. The patient did not present any neurovascular abnormalities in the upper limbs.

Given the clinical suspicion of bilateral glenohumeral dislocation, one ampoule each of pethidine 50 $\mathrm{mg}$ (intramuscular) and diazepam $10 \mathrm{mg}$ (sublingual) was administered. Anteroposterior x-rays on both shoulders revealed the existence of bilateral anterior glenohumeral dislocation, without apparent signs of fracture (Figure 1).

In this light, and with the patient calmer and complaining less after taking the medication, the dislocation was reduced by means of the modified Milch technique, sequentially in both shoulders.

After the reduction, a new neurovascular assessment

1 - Resident Physician in Orthopedics and Traumatology, Alto Minho Local Healthcare Unit, EPE, Porto, Portugal.

2 - Specialist Attending Physician in Orthopedics and Traumatology, Alto Minho Local Healthcare Unit, EPE, Porto, Portugal.

Work performed at the Orthopedics and Traumatology Service, Alto Minho Local Healthcare Unit, EPE, Viana do Castelo, Portugal

Correspondence: Luís Fernando Nunes Pires da Silva - Rua Conde Campo Bello, 105 - $1^{\circ} \mathrm{G}$ - 4200-603 Porto - Portugal. E-mail: medicina.mail@gmail.com

Work received for publication: April 4, 2010; accepted for publication: July 21, 2010. 
was made, and new X-rays were produced to confirm that the joint structures were well positioned and to rule out any possible fracture lines that might have initially been hidden or any intercurrences from the reduction maneuvers (Figure 2).

The patient was then immobilized with the upper limbs in adduction and internal rotation for three weeks. Thereafter, pendular movements were progressively resumed, as tolerated.

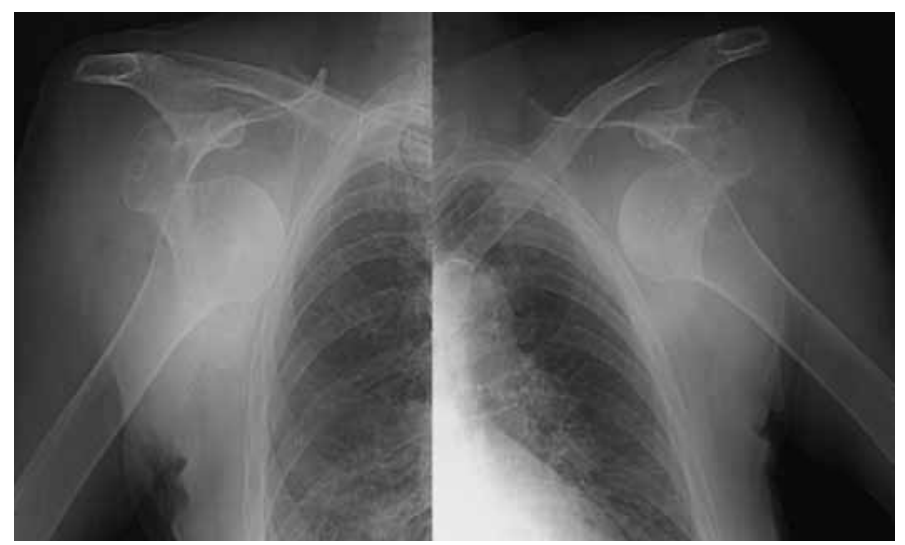

Figure 1 - X-ray images: bilateral anterior dislocation of the shoulders

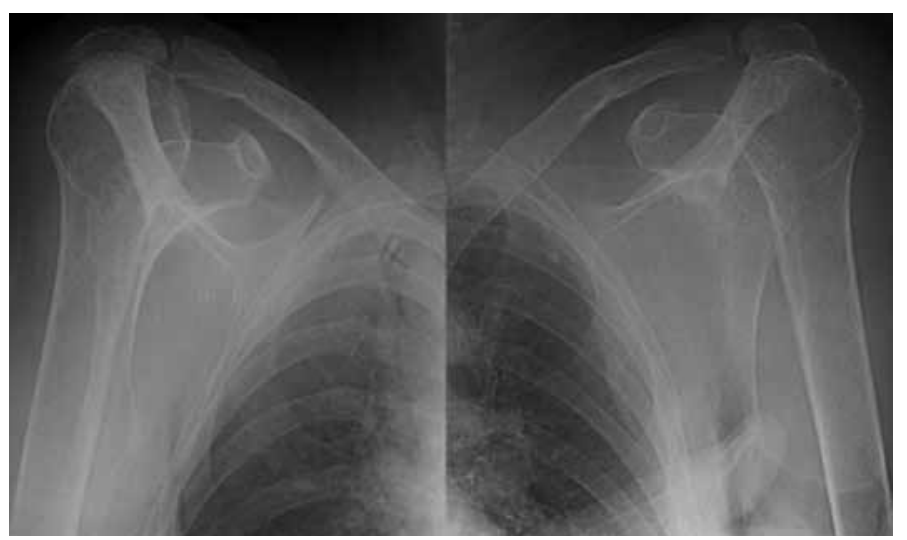

Figure 2 - X-ray images: bilateral anterior dislocation of the shoulders after reduction.

\section{DISCUSSION}

Bilateral glenohumeral dislocation was first described by Mynter ${ }^{(1)}$ in 1902, in patients with camphor poisoning who presented convulsive crises. The combination of bilateral glenohumeral dislocation is a clinical rarity, and posterior bilateral dislocation is the commonest form, followed by the anterior form and lastly, the inferior form (erect dislocation). With regard to etiology, bilateral dislocation may originate from: violent muscle contractions (49\%), which includes convulsive crises, electrocution and physical exercise; traumatic events (23\%), including falls and violent traction on the limbs; and non-traumatic situations (36\%), which include neuromuscular diseases, joint hyperlaxity or voluntary dislocation ${ }^{(2-4)}$. Its strong connection with the existence of convulsive crises (with characteristics violent muscle spasms) has led some authors to state that the presence of bilateral glenohumeral dislocation immediately makes it necessary to conduct a complete neurological and metabolic evaluation in order to rule out unknown pathological conditions from this forum ${ }^{(5)}$. In the literature, fewer than 50 cases of bilateral anterior glenohumeral dislocation have been described, and most of them originated from violent muscle contractions (convulsive crises) or direct trauma ${ }^{(4,6)}$. There are at least four case descriptions of bilateral glenohumeral dislocation in individuals doing muscle training exercises $^{(3)}$.

The forces needed to trigger bilateral glenohumeral dislocation need to be simultaneous and symmetrical $^{(4,7)}$. The commonest mechanism leading to anterior glenohumeral dislocation usually involves forced extension of the upper limb, accompanied by external rotation and abduction. This movement leads to a conflict between the humeral trochiter and the acromion, which acts as the fulcrum of a lever for the dislocation, sometimes in association with fracturing of the trochiter ${ }^{(3,8)}$. In the clinical case presented, the mechanism of dislocation was as described, accompanied by retropulsion caused during the fall when the patient put her weight on her hands, on the ground. Bilateral posterior dislocation is more common in situations of convulsive crises, unlike anterior dislocation, which appears more often during direct trauma. During the violent muscle contractions of convulsive crises or electrocution, because the internal rotators of the humerus are more powerful than the external rotators, this obliges the humerus to undergo maximum internal rotation and adduction, which gives rise to posterior glenohumeral dislocation ${ }^{(6)}$.

Unilateral glenohumeral dislocation is one of the pathological conditions of joints that most often goes unnoticed by orthopedists. Hawkins et $\mathrm{al}^{(9)}$ published a review on 40 cases of unilateral glenohumeral dislocation, in which it was seen that the mean time that elapsed from the initial trauma to the time of the diag- 
nosis was around one year. No inferences about what the situation would be regarding bilateral posterior dislocation were made. In another review article, Dunlop ${ }^{(6)}$ reported that around $10 \%$ of the bilateral anterior dislocations seen by the emergency services remained undiagnosed, or were incompletely diagnosed.

To correctly diagnose glenohumeral dislocation and what its direction is, X-rays have to be produced in two planes before making any attempt at reduction, which preferably should be in the anteroposterior and axillary views. However, producing axillary $\mathrm{X}$-rays is extremely painful for the patient, and it is acceptable to replace this with a scapular (Y) view, at the cost of loss of sensitivity, or if there is some doubt, with a TAC scan on the joint ${ }^{(9,10)}$.

As soon as the correct diagnosis has been established, the joint reduction needs to be performed as rapidly as possible, given the risk of neurovascular impairment and increased difficulty in the maneuver due to establishment of muscle contractures. Although rare, the possibility of neurovascular lesions should always be investigated when cases of suspected glenohumeral dislocation arrive at the emergency services, as well as after the joint reduction, given that there is a real risk that these structures (axillary nerve, musculocutaneous nerve and brachial plexus) may become stretched during the reduction maneuvers $^{(10)}$.

There is still no consensus regarding the protocol for actions after release from the emergency services. However, most authors have advocated immobilization in internal rotation and adduction for as long as the pain lasts (three to four weeks), followed by active mobilization with progressive pendular movements, as tolerated, plus physiotherapy ${ }^{(10)}$. If there are signs of instability or persistent pain, it may be necessary to investigate whether other, associated lesions may be present.

\section{REFERENCES}

1. Mynter H. Subacromial dislocation from muscular spasm. Ann Surg. 1902;36:117-9.

2. Brown RJ. Bilateral dislocation of the shoulders. Injury. 1984;15(4):267-73.

3. Felderman H, Shih R, Maroun V. Chin-up-induced bilateral anterior shoulder dislocation: a case report. J Emerg Med. 2009;37(4):400-2.

4. Abalo A, Dossim A, Songné B, Ayouba G. Luxation antérieure bilateral desépaules - à propos d'um cas. Chir Main. 2008;27(2-3):118-21.

5. Elliott DS. Brain tumor presenting as a dislocated shoulder. J Bone Joint Surg Br. 1990;72(5):925.
6. Dunlop CC. Bilateral anterior shoulder dislocation - case report and review of the literature. Acta Orthop Belg. 2002;68(2):168-70.

7. Cresswell TR, Smith RB. Bilateral anterior shoulder dislocations in bench pressing: an unusual case. Br J Sports Med. 1998;32(1):71-2.

8. Siwach R, Singh R, Rohilla R. Bilateral anterior dislocation of the shoulder - a case report and review of the literature. Injury Extra. 2008;39:394-7.

9. Hawkins RJ, Neer CS 2nd, Pianta RM, Mendoza FX. Locked posterior dislocation of the shoulder. J Bone Joint Surg Am. 1987;69(1):9-18.

10. Wen DY. Current concepts in the treatment of anterior shoulder dislocations. Am J Emerg Med. 1999;17(4):401-7. 